\title{
GLOBAL FINITE GENERATING FUNCTIONS FOR FIELD THEORY
}

\author{
FRANCO CARDIN \\ Dipartimento di Matematica Pura ed Applicata, Università di Padova \\ via Belzoni, 7, 35131 Padova, Italy \\ E-mail: cardin@math.unipd.it
}

\begin{abstract}
We introduce an infinite-dimensional version of the Amann-Conley-Zehnder reduction for a class of boundary problems related to nonlinear perturbed elliptic operators with symmetric derivative. We construct global generating functions with finite auxiliary parameters, describing the solutions as critical points in a finite-dimensional space.
\end{abstract}

1. Introduction. Research in symplectic topology applied to mechanics has been highly developed in the last two decades, mainly due to Arnol'd, Chaperon, Sikorav, Viterbo and other authors. For example, a very important conjecture (Weinstein) on the Calculus of Variations has been resolved by Viterbo [V1] in 1987 by using modern topological symplectic techniques; he was able to show - as a consequence of a more general theorem - that every compact energy surface admits at least one periodic orbit. These techniques, arising on the topological side from Morse and Lusternik-Schnirelman theory, and on the symplectic side from early works by Maslov and Hörmander in the sixties, were developed e.g. by Libermann, Lichnerowicz, Marle, Weinstein and other authors in geometry, but surely their crucial role in analytical mechanics has been exhaustively investigated by Tulczyjew, see e.g. [T2], [T3]. Among many interesting applications and results, we may recall the fruitful use of the symplectic arena in the theory of the Hamilton-Jacobi equation, both in the geometrical mechanical framework - see the seminal paper $[\mathrm{T} 1]$ and the paper $[\mathrm{B}-\mathrm{T}]$ - and as well as in the purely analytical one: by using the global Hamilton principal function with parameters, Bardi and Evans [B-E] in 1984 were able, first, to give an explicit construction for the viscosity solution of a H-J problem (i.e., $\partial S / \partial t+H(\partial S / \partial x)=0, S(0, x)=\sigma(x))$, for a $p$-convex Hamiltonian $H(p)$,

2000 Mathematics Subject Classification: Primary 53D12; Secondary 49J35.

Key words and phrases: symplectic geometry, calculus of variations.

This paper has been performed in the sphere of the activities supported by the G.N.F.M.I.N.d'A.M. and by the M.I.U.R. of Italy.

The paper is in final form and no version of it will be published elsewhere. 
by means of an inf-sup procedure on the auxiliary parameters, an obvious - although non trivial - analytical version of the well known stationarization method to describe the related Lagrangian submanifold, the geometrical solution of the same problem. Almost ten years ago, Chaperon and Viterbo, see [V3], proposed a new global theory of solutions for H-J equations: the so-called min-max solution, founded on symplectic topology and the theory of the Lusternik-Schnirelman. Here one encounters a very intriguing fact, not yet completely understood: sometimes, when e.g. the Hamiltonian function is not $p$-convex, the viscosity solution and the min-max solution do not coincide.

In [V2], Viterbo proposed a version of the Amann-Conley-Zehnder Reduction (see [A-Z]) in order to construct global generating functions of the (wave-front) geometrical solutions of H-J problems of the evolution type in the non-compact environment $Q=R^{n}$. Whenever these generating functions are 'quadratic at infinity'-here we do not enter into details - then they are Palais-Smale functions, so the Lusternik-Schnirelman theory works in order to find the min-max solutions. These techniques have recently been taken into account and utilized to build global generating functions of the (space-time) geometrical solutions for the above H-J problems [C3], a result well known whenever the Hamiltonian system is integrable, see [T1], [C1], [C2].

In this paper, in Sec. 2, we recall the Amann-Conley-Zehnder reduction scheme in Viterbo's version and we construct the afore mentioned global generating function for the wave-front Lagrangian submanifold of a H-J problem starting from the zero section. In Sec. 3, we consider a simple infinite-dimensional system (Neumann boundary problem for a scalar harmonic field) and we recall the (more or less standard) interpretation of the functional of its variational formulation as a generating function with infinite auxiliary parameters. In Sec. 4, we consider a certain class of infinite-dimensional nonlinear problems related to operators with symmetric derivative, having the structure of nonlinear perturbation of an elliptic problem. Motivated by the above Amann-Conley-Zehnder reduction, we construct global generating functions with finite parameters, describing the solutions as critical points in a finite-dimensional space. (These techniques seems to be comparable with a Liapunov-Schmidt reduction, although this last procedure is rather different.) Notice now that this theory allows us to study the existence of solutions by searching for critical points of a function defined on a finite-dimensional space: if this function is Palais-Smale, the search can be performed, as already fruitfully done in related subjects, by techniques from the Lusternik-Schnirelman theory.

I gratefully thank Włodzimierz Marek Tulczyjew, whose relevant experience has been transmitted during many enriching and illuminating conversations which allowed the maturation of several geometrical ideas of this and other papers.

\section{Global generating functions with finite auxiliary parameters for evolu- tive Hamilton-Jacobi problems}

2.1. The Hamilton-Helmholtz functional. In this section we sketch an application of the Amann-Conley-Zehnder reduction, in Viterbo's version, in order to see the construction of global generating functions describing the Lagrangian wave-front set (at the time $t=T>0)$ related to the evolutive Hamilton-Jacobi equation and starting at $t=0$ 
(for simplicity, and without loss of generality) from the zero-section $\Lambda_{0}$ of $T^{*} R^{n}, \Lambda_{0}=$ $\left\{(q, p) \in T^{*} R^{n}: q \in R^{n}, p=0\right\}$ :

$$
\frac{\partial S}{\partial t}(q, t)+H\left(t, q, \frac{\partial S}{\partial q}(q, t)\right)=0,\left.\quad S(q, t)\right|_{t=0} \equiv 0 .
$$

Let us consider the set of curves

$$
\Gamma:=\left\{\gamma(\cdot)=(q(\cdot), p(\cdot)) \in H^{1,2}\left([0, T], R^{2 n}\right): p(0)=0\right\} .
$$

By the Sobolev imbedding theorem, $H^{1,2}\left((0, T), R^{2 n}\right) \hookrightarrow \mathcal{C}^{0}\left([0, T], R^{2 n}\right)$, and it is selfevident that the set $\Gamma$ has a natural linear space structure, and then $T_{\gamma} \Gamma=\Gamma$, for all $\gamma \in \Gamma$.

An equivalent way of describing the curves of $\Gamma$ is to assign the $q$-projection of the end point, $q(T) \in R^{n}$, and the velocity $\dot{\gamma}$, for each $s \in[0, T]$, of the curve $\gamma$ by means of a function $\phi \in L^{2}$. One then has the following simple

LEMma 1. For all $\phi \in L^{2}$ set $\phi=\left(\phi_{q}, \phi_{p}\right)$. The map $g$,

$$
g: R^{n} \times L^{2} \rightarrow \Gamma, \quad(q(T), \phi) \mapsto g(q(T), \phi)(s):=\left(q(T)-\int_{s}^{T} \phi_{q}(r) d r, \int_{0}^{s} \phi_{p}(r) d r\right)
$$

is a bijection.

We recall that the flow $\Phi^{s}=\Phi_{X_{H}}^{s, 0}, \Phi_{X_{H}}^{0,0}=\mathrm{id}_{R^{2 n}}$, related to Hamilton's equations $\dot{\gamma}=J \nabla H$, transforms Lagrangian manifolds into Lagrangian manifolds: $\Lambda_{s}=\Phi^{s}\left(\Lambda_{0}\right)$. We call $\Lambda=\Lambda_{T}=\Phi^{T}\left(\Lambda_{0}\right)$ the Lagrangian wave-front set at the time $T>0$.

The map

$$
A: \Gamma \rightarrow R, \quad \gamma \mapsto A[\gamma]:=\int_{0}^{T}[p(s) \cdot \dot{q}(s)-H(s, q(s), p(s))] d s
$$

is the action functional of the Hamilton-Helmholtz variational principle related to the Hamiltonian $H$. By a simple direct computation, the following lemma allows us to regard the action functional $A$ as a generating function of $\Lambda_{T}$ with infinite parameters (in $L^{2}$ ).

LEMMA 2.2. The map

$$
W:=A \circ g: R^{n} \times L^{2} \rightarrow R, \quad(q(T), \phi) \mapsto W(q(T), \phi):=A \circ g(q(T), \phi)=A[g(q(T), \phi)]
$$
generates $\Lambda_{T}$, that is:

$$
\Lambda_{T}=\left\{(q(T), p(T)): q(T) \in R^{n}, \quad p(T)=\frac{\partial W}{\partial q(T)}(q(T), \phi), \quad \frac{D W}{D \phi}(q(T), \phi)=0\right\} .
$$

2.2. Cut-off decomposition of Fourier expansions and fixed point. For every $\phi \in L^{2}$ let us consider the Fourier expansion

$$
\phi(s)=\sum_{k \in Z} \phi_{k} e^{i \frac{2 \pi k}{T} s} .
$$

For each fixed $m \in N$ let us consider the projection maps on the basis $\left\{e^{i \frac{2 \pi k}{T} s}\right\}_{k \in Z}$ of $L^{2}$,

$$
P_{m} \phi(s):=\sum_{|k| \leq m} \phi_{k} e^{i \frac{2 \pi k}{T} s}, \quad Q_{m} \phi(s):=\sum_{|k|>m} \phi_{k} e^{i \frac{2 \pi k}{T} s} .
$$

Clearly,

$$
P_{m} L^{2} \oplus Q_{m} L^{2}=L^{2},
$$

and for $\phi \in L^{2}$ we will write $u:=P_{m} \phi$ and $v:=Q_{m} \phi$. 
REMARK 2.1. The idea for proving existence of a generating function with finitely many parameters is to show that the 'infinite' tail $Q_{m} \dot{\gamma}$ of $\dot{\gamma}$, for $\gamma$ a curve in $\Gamma$ solving Hamilton's equations, can be deleted from the expression of the action functional; in other words, $\gamma$ is completely determined by a suitable choice of its 'finite' part $P_{m} \dot{\gamma}$, for suitable (large) $m \in N$.

Lemma 2.3 (Lipschitz). For fixed $q(T) \in R^{n}$ and $u \in P_{m} L^{2}$, the map

$$
Q_{m} L^{2} \rightarrow\left(\Gamma,\|\cdot\|_{L^{2}}\right), \quad v \mapsto g(q(T), u+v)
$$

is Lipschitz with

$$
\operatorname{Lip}(g) \leq \frac{T}{2 \pi m}(1+\sqrt{2 m}) .
$$

LEMMA 2.4 (Contraction map). Suppose that

$$
\sup _{y \in R^{2 n}, s \in[0, T]}\left|\nabla_{y y}^{2} H\right|=C<+\infty \quad(y:=(q, p)) .
$$

For $m$ large enough:

$$
\frac{T C}{2 \pi m}(1+\sqrt{2 m})<1
$$

for every fixed $q(T) \in R^{n}$ and $u \in P_{m} L^{2}\left([0, T] ; R^{2 n}\right)$ the map:

$$
Q_{m} L^{2}\left([0, T] ; R^{2 n}\right) \rightarrow Q_{m} L^{2}\left([0, T] ; R^{2 n}\right), \quad v \mapsto Q_{m} J \nabla H(g(q(T), u+v))
$$

is a contraction map.

By the Banach-Caccioppoli Theorem there exists one and only one fixed point $f(q(T), u)$ for the above contraction map. By standard arguments one can easily see that this fixed point depends smoothly on $q(T)$ and $u$, and one has

$$
f(q(T), u)=Q_{m} J \nabla H(g(q(T), u+f(q(T), u))) .
$$

It is crucial to observe that, if we solve the finite (say, algebraic) equation for $u$,

$$
u=P_{m} J \nabla H(g(q(T), u+f(q(T), u))),
$$

and we sum the last two formulas, then the resulting equation

$$
\dot{\gamma}=J \nabla H(\gamma)
$$

implies that the curve $\gamma=g(q(T), u+f(q(T), u))$ solves the Hamilton canonical differential equations, and it starts from the zero section (so that $\gamma \in \Gamma$ ). Furthermore, we point out that $\operatorname{dim}\left(P_{m} L^{2}\left([0, T] ; R^{2 n}\right)\right)=2 n(2 m+1)=: k(n, m)$.

To conclude, we must establish the following Theorem.

THEOREM 2.1 (The finite-parameters generating function). The function

$$
\begin{gathered}
S: R^{n} \times R^{k(n, m)} \rightarrow R, \\
(q(T), u) \mapsto S(q(T), u):=A \circ g(q(T), u+f(q(T), u))=W(q(T), u+f(q(T), u)),
\end{gathered}
$$

is a global generating function for $\Lambda=\Phi^{T}\left(\Lambda_{0}\right)$.

Proof. We write

$$
\frac{\partial S}{\partial u}(q(T), u)=\frac{D W}{D \phi}\left(\frac{D \phi}{D u}+\frac{D \phi}{D v} \frac{D f}{D u}\right)
$$


(note that $\frac{D \phi}{D u}$ and $\frac{D \phi}{D v}$ are the projectors $P_{m}$ and $Q_{m}$ respectively),

$$
\begin{aligned}
\frac{\partial S}{\partial u}(q(T), u)= & -\left.\int_{0}^{T}\left[P_{m}(J \dot{\gamma}+\nabla H(\gamma))\right]\right|_{\gamma=g(q(T), u+f(q(T), u))} d s \\
& -\left.\int_{0}^{T}\left[Q_{m}(J \dot{\gamma}+\nabla H(\gamma))\right]\right|_{\gamma=g(q(T), u+f(q(T), u))} \frac{D f}{D u} d s .
\end{aligned}
$$

By the very construction of $f(q(T), u)$ the second integral vanishes, so

$$
\frac{\partial S}{\partial u}(q(T), u)=-\left.\int_{0}^{T}\left[P_{m}(J \dot{\gamma}+\nabla H(\gamma))\right]\right|_{\gamma=g(q(T), u+f(q(T), u))} d s,
$$

so that

$$
\frac{\partial S}{\partial u}(q(T), u)=0 \quad \text { is equivalent to }\left.\quad\left[P_{m}(J \dot{\gamma}+\nabla H(\gamma))\right]\right|_{\gamma=g(q(T), u+f(q(T), u))}=0 .
$$

On the other hand,

$$
\begin{gathered}
\frac{\partial S}{\partial q(T)}=\frac{\partial W}{\partial q(T)}+\frac{D W}{D \phi} \frac{D \phi}{D v} \frac{D f}{D q(T)} \\
\frac{\partial S}{\partial q(T)}=\frac{\partial W}{\partial q(T)}-\left.\int_{0}^{T}\left[Q_{m}(J \dot{\gamma}+\nabla H(\gamma))\right]\right|_{\gamma=g(q(T), u+f(q(T), u))} \frac{D f}{D q(T)} d s
\end{gathered}
$$

hence

$$
\frac{\partial S}{\partial q(T)}(q(T), u)=\left.\frac{\partial W}{\partial q(T)}(q(T), \phi)\right|_{\phi=u+f(q(T), u)} .
$$

Now it is easy to conclude that the pair $(q(T), \phi) \in R^{n} \times L^{2}$ satisfies

$$
\left\{\begin{aligned}
p(T) & =\frac{\partial W}{\partial q(T)}(q(T), \phi), \\
0 & =\frac{D W}{D \phi}(q(T), \phi),
\end{aligned}\right.
$$

if and only if the pair $(q(T), u) \in R^{n} \times R^{k(n, m)}$, where

$$
\phi=u+f(q(T), u), \quad \text { so that } u=P_{m} \phi,
$$

satisfies

$$
\left\{\begin{aligned}
p(T) & =\frac{\partial S}{\partial q(T)}(q(T), u) \\
0 & =\frac{\partial S}{\partial u}(q(T), u) .
\end{aligned}\right.
$$

3. Global generating functions with infinite auxiliary parameters for linear elliptic problems. In this section we interpret the functional $J$ of the calculus of variations for the Neumann boundary problem of the Laplace equation as a generating function with "infinite parameters". It describes a Lagrangian set drawing the physical landscape related to the solutions of the problem.

In a bounded Stokesian set $\Omega \subset R^{n}$ we consider a suitable space $H$ of functions $u: \Omega \rightarrow R$ (e.g., $H^{1,2}$ ) and we take into account also a space of functions defined on the boundary of $\Omega, B=B^{\partial \Omega}, q(\cdot) \in B, q: \partial \Omega \rightarrow R$. We denote by $T^{*} B \cong B \times B$ the (formal) cotangent bundle of $B$ and the (candidate) generating function:

$$
J: B \times H \rightarrow R, \quad(q, u) \mapsto J(q, u):=\frac{1}{2} \int_{\Omega}|\nabla u|^{2}-\int_{\partial \Omega} q u .
$$


We perform the following computation, in the sense of distributions:

$$
\begin{aligned}
\frac{\partial J}{\partial u}(q, u) h & =\int_{\Omega} \nabla u \cdot \nabla h-\int_{\partial \Omega} q h \\
& =\int_{\Omega}[\nabla \cdot(\nabla u h)-\Delta u h]-\int_{\partial \Omega} q h, \\
& =-\int_{\Omega} \Delta u h+\int_{\partial \Omega}(\nabla u \cdot n-q) h .
\end{aligned}
$$

Thus,

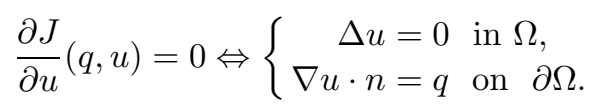

Now we define

$$
p=\frac{\partial J}{\partial q}(q, u) \psi=\int_{\partial \Omega} u \psi .
$$

Finally, the Lagrangian set $\Lambda \subset T^{*} B$, which collects all the solutions of the Neumann problems, is precisely defined by the generating function with infinite auxiliary parameters $u \in H$ given by the functional $J$ :

$$
\Lambda=\left\{(q, p) \in T^{*} B: p=\frac{\partial J}{\partial q}(q, u), \quad 0=\frac{\partial J}{\partial u}(q, u)\right\} .
$$

\section{Global finite generating functions for nonlinear variational elliptic prob- lems}

4.1. Volterra-Vainberg systems. As in Sec. 3, let $\Omega \subset R^{n}$ be a Stokesian set, and consider a suitable space $H$ of functions $u$,

$$
u: \Omega \ni x \mapsto u(x) \in R^{k} .
$$

Later we will specify the structure of the function space. Consider a nonlinear operator

$$
N: H \rightarrow K \text {. }
$$

For example, by thinking of a context like elasticity, setting

i) the function spaces:

$$
H=C^{\infty}\left(\bar{\Omega} ; R^{k}\right), \quad K=C^{\infty}\left(\bar{\Omega} ; R^{k}\right) \times C^{\infty}\left(\partial \Omega ; R^{k}\right),
$$

ii) $\mathcal{N}(u)$ : the 'divergence' operator acting on the stress, depending on $\nabla u$ (so that $\mathcal{N}(u)$ is a second order differential operator), and

iii) for fixed $q \in C^{\infty}\left(\partial \Omega ; R^{k}\right)$,

we define the operator

$$
H \ni u \mapsto N(u):=\left(\mathcal{N}(u), \nabla_{x} u \cdot n-q\right) \in K,
$$

so the nonlinear equation $N(u)=0$ is a (version of the) Neumann boundary problem on the domain $\Omega$.

Suppose the derivative of $N$ is symmetric with respect to a nondegenerate bilinear form $\langle\rangle:, K \times H \rightarrow R$,

$$
\left\langle N^{\prime}(u) h, k\right\rangle=\left\langle N^{\prime}(u) k, h\right\rangle \quad \forall u, h, k \in H,
$$


where, for example,

$$
\left\langle\left(v_{1}, v_{2}\right), u\right\rangle=\int_{\Omega} v_{1} \cdot u d^{(n)} x+\int_{\partial \Omega} v_{2} \cdot u d^{(n-1)} x .
$$

In this case, by invoking the Volterra-Vainberg theorem [Vo], [Va], the above equation $N(u)=0$ is equivalent to the following variational principle:

$$
d J[u] h=0, \quad \forall h \in H,
$$

where

$$
J[u]:=\int_{0}^{1}\langle N(t u), u\rangle d t .
$$

Indeed:

$$
\begin{aligned}
J^{\prime}[u] h=\frac{d}{d \lambda} J & \left.(u+\lambda h)\right|_{\lambda=0}=\int_{0}^{1}\left[\left\langle N^{\prime}(t u) t h, u\right\rangle+\langle N(t u), h\rangle\right] d t \\
& =\int_{0}^{1}\left[\left\langle N^{\prime}(t u) u, t h\right\rangle+\langle N(t u), h\rangle\right] d t=\int_{0}^{1} \frac{d}{d t}[\langle N(t u), t h\rangle] d t=\langle N(u), h\rangle .
\end{aligned}
$$

4.2. Nonlinear perturbation of elliptic problems. Fix a function $\bar{u}$ satisfying $\nabla \bar{u} \cdot n=q$ on $\partial \Omega$. We will search for functions $w$ satisfying $\mathcal{N}(\bar{u}+w)=0$ in $\Omega$ with $\nabla w \cdot n=0$ on $\partial \Omega$. Equivalently, we may consider the above boundary problem with $q=0$, since we have seen this is not restrictive.

Let us now suppose that the structure of $\mathcal{N}(u)$ is of the form $-L u+F(\varepsilon, u)$, or, simply,

$$
\mathcal{N}(u)=-L u+\varepsilon F(u),
$$

where $L$ is a linear elliptic operator, and $F$ a nonlinear operator, both mapping $H=$ $C^{\infty}\left(\bar{\Omega} ; R^{k}\right)$ into itself. Our main hypothesis is that $L$ and $F^{\prime}$ are symmetric with respect to $\langle$,$\rangle ; moreover, we will suppose ellipticity for L$ on $H=C^{\infty}\left(\bar{\Omega} ; R^{k}\right)$ with $\nabla u \cdot n=0$ :

$$
-L u_{j}=\lambda_{j} u_{j}, \quad \nabla u_{j} \cdot n=0, \quad\left\langle u_{i}, u_{j}\right\rangle_{L^{2}}=\delta_{i j}, \quad \lambda_{0}=0<\lambda_{1} \leq \lambda_{2} \leq \ldots
$$

so that the related linear problem

$$
L u=f,
$$

for generic $f \in H$ with $\nabla f \cdot n=0$, is solved in a unique way by

$$
u=g(f):=-\sum_{j=1}^{+\infty} \frac{u_{j}}{\lambda_{j}}\left\langle f, u_{j}\right\rangle_{L^{2}} .
$$

4.3. Cut-off decomposition of $H$. Given a natural number $m \in N$, we define the following decomposition of $H$ :

$$
\begin{gathered}
\mu=P_{m} u:=\sum_{j=1}^{m} u_{j}\left\langle u, u_{j}\right\rangle_{L^{2}}, \quad \eta=Q_{m} u:=\sum_{j>m}^{+\infty} u_{j}\left\langle u, u_{j}\right\rangle_{L^{2}}, \\
H=P_{m} H \oplus Q_{m} H, \quad u=\mu+\eta .
\end{gathered}
$$

Now, fix $m \in N, \mu \in P_{m} H$, and consider the map

$$
\tilde{g}: Q_{m} H \rightarrow H, \quad \eta \mapsto \tilde{g}(\eta):=g(\mu+\eta) .
$$


We recognize that this map $\eta \mapsto \tilde{g}(\eta)$ is Lipschitz:

$$
\left|\tilde{g}\left(\eta_{2}\right)-\tilde{g}\left(\eta_{1}\right)\right|=\left|\sum_{j>m}^{+\infty} \frac{u_{j}}{\lambda_{j}}\left\langle\eta_{2}-\eta_{1}, u_{j}\right\rangle_{L^{2}}\right| \leq \frac{1}{\lambda_{m}}\left|\eta_{2}-\eta_{1}\right| .
$$

4.4. The fixed point map. Finally, once again for fixed $m \in N, \mu \in P_{m} H$, consider the map

$$
Q_{m} H \rightarrow Q_{m} H, \quad \eta \mapsto \varepsilon Q_{m}(F(g(\mu+\eta))) .
$$

This map, under the hypothesis that

$$
\sup _{u \in H}\left|F^{\prime}(u)\right| \leq C<+\infty
$$

is a contraction map if for a suitably small perturbation parameter $\varepsilon$, and for a suitably large cut-off number $m$, we have:

$$
\frac{\varepsilon C}{\lambda_{m}}<1
$$

Indeed:

$$
\left|\varepsilon Q_{m}\left(F\left(g\left(\mu+\eta_{2}\right)\right)\right)-\varepsilon Q_{m}\left(F\left(g\left(\mu+\eta_{1}\right)\right)\right)\right| \leq \frac{\varepsilon C}{\lambda_{m}}\left|g\left(\eta_{2}\right)-g\left(\eta_{1}\right)\right| .
$$

The Banach-Caccioppoli theorem gives us a unique fixed point $\mathrm{d}(m, \mu)$ for this contraction map, it works in the following way:

$$
\mathrm{d}(m, \mu)=\varepsilon Q_{m}(F(g(\mu+\mathrm{d}(m, \mu)))) .
$$

Together with $(*)$, we consider the finite equation for $\mu \in P_{m} H \cong R^{m+1}$ :

$$
\mu=\varepsilon P_{m}(F(g(\mu+\mathrm{d}(m, \mu)))) .
$$

Concretely, in connection with each solution $\mu$ of $(* *)$, we note that, by adding $(*)$ to $(* *)$, we obtain one (and only one) solution of the nonlinear problem $L u=\varepsilon F(u), \nabla u \cdot n=0$, by the formula:

$$
u=g(\mu+\mathrm{d}(m, \mu))
$$

REMARK 4.1. It is interesting to investigate what happens when one considers the finite equation $(* *)$ in a sequence of spaces $R^{m}$ of increasing dimension. Let us initially consider $m=1$, so we are led to take into account rather small values for $\varepsilon$ :

$$
0 \leq \varepsilon<\lambda_{1} / C, \quad \mu \in R^{1}
$$

Whenever we wish to consider perturbations $\varepsilon \geq \lambda_{1} / C$, we should take $m$ to be at least 2 :

$$
\lambda_{1} \leq \lambda_{2}, \quad \varepsilon<\lambda_{2} / C, \quad \mu \in R^{2} .
$$

We notice that, for a fixed $\varepsilon<\lambda_{1} / C$, the set of the solutions $u=g(\mu+\mathrm{d}(1, \mu))$ generated by the equation in $R^{1}$

$$
\mu=\varepsilon P_{1}(F(g(\mu+d(1, \mu)))), \quad \varepsilon<\lambda_{1} / C,
$$

is contained in the set of the solutions $u=g(\mu+\mathrm{d}(2, \mu))$ generated by the equation in $R^{2}$

$$
\mu=\varepsilon P_{2}(F(g(\mu+\mathrm{d}(2, \mu)))), \quad \varepsilon<\lambda_{1} / C .
$$

When $\varepsilon$ crosses the value $\lambda_{1} / C$, this last equation in $R^{2}$ could suddenly produce a growing number of solutions; in other words, we can recognize in the following sequence of 'critical' 
perturbation values

$$
\varepsilon=\lambda_{1} / C, \quad \lambda_{2} / C, \quad \lambda_{3} / C, \ldots
$$

a sequence of possible approximate ( $C$ may not be optimal) bifurcation values for the global $\varepsilon$-depending set of solutions of our variational problem $L u=\varepsilon F(u)$, indicating a hierarchically growing complexity for it.

4.5. Global generating function with a finite number of auxiliary parameters. For a fixed $m \in N$, we define the real-valued function $W: R^{m} \rightarrow R$ by

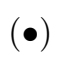

$$
W(\mu):=J \circ g(\mu+\mathrm{d}(m, \mu)) .
$$

THEOREM 4.1. Suppose that

$$
\sup _{\phi \in H}\left|F^{\prime}(\phi)\right| \leq C<+\infty .
$$

Then, for fixed $\varepsilon<\lambda_{m} / C$, the stationary points of $W: R^{m} \rightarrow R$, say $\mu_{1}, \ldots, \mu_{\alpha}, \ldots, \mu_{n_{m}}$, produce exactly the stationary points of $J$ :

$$
u_{1}=g\left(\mu_{1}+\mathrm{d}\left(m, \mu_{1}\right)\right), \ldots, u_{\alpha}=g\left(\mu_{\alpha}+\mathrm{d}\left(m, \mu_{\alpha}\right)\right), \ldots, u_{n_{m}}=g\left(\mu_{n_{m}}+\mathrm{d}\left(m, \mu_{n_{m}}\right)\right) .
$$

Proof.

$$
\begin{aligned}
d W & =\frac{\partial W}{\partial \mu}(\mu) d \mu=\left.d J[u]\right|_{u=g(\mu+\mathrm{d}(m, \mu))} d_{\mu} g(\mu+\mathrm{d}(m, \mu)), \\
& =\left\langle\left.(L u-\varepsilon F(u))\right|_{u=g(\mu+\mathrm{d}(m, \mu))}, d_{\mu} g(\mu+\mathrm{d}(m, \mu))\right\rangle,
\end{aligned}
$$

but from the definition of the map $g$ and from the very definition $(*)$ of the fixed point $\mathrm{d}(m, \mu)$ we have

$$
L(g(\mu+\mathrm{d}(m, \mu)))=\mu+\mathrm{d}(m, \mu), \quad Q_{m}(\mu+\mathrm{d}(m, \mu)-\varepsilon F(g(\mu+\mathrm{d}(m, \mu))))=0,
$$

hence

$$
\left.Q_{m}(L u-\varepsilon F(u))\right|_{u=g(\mu+\mathrm{d}(m, \mu))}=0,
$$

thus

$$
d W=\left\langle\mu-\varepsilon P_{m} F(g(\mu+\mathrm{d}(m, \mu))), P_{m} d_{\mu} g(\mu+\mathrm{d}(m, \mu))\right\rangle .
$$

From $g(f)=-\sum_{j>0}^{+\infty} \frac{u_{j}}{\lambda_{j}}\left\langle f, u_{j}\right\rangle_{L^{2}}$, it is easy to see that

$$
P_{m} d_{\mu} g(\mu+\mathrm{d}(m, \mu))=-\sum_{j>0}^{m} \frac{u_{j}}{\lambda_{j}} d \mu_{j},
$$

so that $d W=0$ if and only if

$$
\mu-\varepsilon P_{m} F(g(\mu+\mathrm{d}(m, \mu)))=0,
$$

which is precisely the condition $(* *)$.

\section{References}

[A-Z] H. Amann and E. Zehnder, Periodic solutions of asymptotically linear hamiltonian systems, Manuscripta Math. 32 (1980), 149-189. 
[B-E] M. Bardi and C. Evans, On Hopf's formulas for solutions of Hamilton-Jacobi equations, Nonlinear Anal. 8 (1984), 1373-1389.

[B-T] S. Benenti and W. M. Tulczyjew, The geometric meaning and globalization of the Hamilton-Jacobi method, in: Differential Geometric Methods in Mathematical Physics (Aix-enProvence/Salamanca, 1979), Lecture Notes in Math. 836, Springer, Berlin, 1980, 9-21.

[C1] F. Cardin, On the geometric Cauchy problem for the Hamilton-Jacobi equation, Nuovo Cimento Sect. B 104 (1989), 525-544.

[C2] F. Cardin, On viscosity and the geometric solutions for the Hamilton-Jacobi equations, Nonlinear Anal. 6 (1993), 713-719.

[C3] F. Cardin, The global finite structure of generic envelope loci for Hamilton-Jacobi equations, J. Math. Phys., 2002, to appear.

[L-M] P. Libermann and C. M. Marle, Symplectic Geometry and Analytical Mechanics, transl. from the French by B. E. Schwarzbach, Math. Appl. 35, Reidel, Dordrecht, 1987.

[M] V. P. Maslov, Théorie des perturbations et méthodes asymptotiques, Editions de l'Université de Moscou, 1965 (in Russian); traduction française: Dunod-Gauthier-Villars, Paris, 1971.

[T1] W. M. Tulczyjew, Relations symplectiques et les équations d'Hamilton-Jacobi relativistes, C. R. Acad. Sc. Paris 281 (1975) 545-547.

[T2] W. M. Tulczyjew, The Legendre transformation, Ann. Inst. H. Poincaré 27 (1977), 101114.

[T3] W. M. Tulczyjew, Geometric Formulations of Physical Theories. Statics and Dynamics of Mechanical Systems, Monographs and Textbooks in Physical Science, Lecture Notes 11, Bibliopolis, Naples, 1989.

[Va] M. M. Vainberg, Variational Methods for the Study of Nonlinear Operators, Holden-Day, San Francisco, 1964.

[V1] C. Viterbo, A proof of Weinstein's conjecture in $R^{2 n}$, Ann. Inst. H. Poincaré Anal. Non Linéaire 4 (1987), 337-356.

[V2] C. Viterbo, Recent progress in periodic orbits of autonomous Hamiltonian systems and applications to symplectic geometry. in: Nonlinear Functional Analysis (Newark, NJ, 1987), Lecture Notes in Pure and Appl. Math. 121, Dekker, New York, 1990, 227-250.

[V3] C. Viterbo, Solutions of Hamilton-Jacobi equations and symplectic geometry. Addendum to: Séminaire sur les Équations aux Dérivés Partielles, 1994-1995, École Polytech., Palaiseau, 1996.

[Vo] V. Volterra, Leçons sur les Fonctions de Ligne, Gauthier-Villars, Paris, 1913. 